\title{
Black carbon fractal morphology and short-wave radiative impact: a modelling study
}

\author{
M. Kahnert and A. Devasthale \\ Swedish Meteorological and Hydrological Institute, 60176 Norrköping, Sweden \\ Received: 5 August 2011 - Published in Atmos. Chem. Phys. Discuss.: 16 August 2011 \\ Revised: 11 November 2011 - Accepted: 14 November 2011 - Published: 24 November 2011
}

\begin{abstract}
We investigate the impact of the morphological properties of freshly emitted black carbon aerosols on optical properties and on radiative forcing. To this end, we model the optical properties of fractal black carbon aggregates by use of numerically exact solutions to Maxwell's equations within a spectral range from the UVC to the mid-IR. The results are coupled to radiative transfer computations, in which we consider six realistic case studies representing different atmospheric pollution conditions and surface albedos. The spectrally integrated radiative impacts of black carbon are compared for two different fractal morphologies, which brace the range of recently reported experimental observations of black carbon fractal structures. We also gauge our results by performing corresponding calculations based on the homogeneous sphere approximation, which is commonly employed in climate models. We find that at top of atmosphere the aggregate models yield radiative impacts that can be as much as 2 times higher than those based on the homogeneous sphere approximation. An aggregate model with a low fractal dimension can predict a radiative impact that is higher than that obtained with a high fractal dimension by a factor ranging between 1.1-1.6. Although the lower end of this scale seems like a rather small effect, a closer analysis reveals that the single scattering optical properties of more compact and more lacy aggregates differ considerably. In radiative flux computations there can be a partial cancellation due to the opposing effects of different error sources. However, this cancellation effect can strongly depend on atmospheric conditions and is therefore quite unpredictable. We conclude that the fractal morphology of black carbon aerosols and their fractal parameters can have a profound impact on their radiative forc-
\end{abstract}

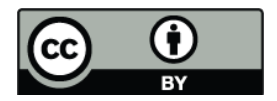

Correspondence to: M. Kahnert (michael.kahnert@smhi.se) ing effect, and that the use of the homogeneous sphere model introduces unacceptably high biases in radiative impact studies. We emphasise that there are other potentially important morphological features that have not been addressed in the present study, such as sintering and coating of freshly emitted black carbon by films of organic material. Finally, we found that the spectral variation of the absorption cross section of black carbon significantly deviates from a simple $1 / \lambda$ scaling law. We therefore discourage the use of single-wavelength absorption measurements in conjunction with a $1 / \lambda$ scaling relation in broadband radiative forcing simulations of black carbon.

\section{Introduction}

Black carbon (BC) imposes a strong positive radiative forcing on the atmosphere due to its high absorption cross section. Among all anthropogenic forcing agents, the contribution of $\mathrm{BC}$ to the total, global direct positive forcing is estimated to be the second largest after that of carbon dioxide (Ramanathan and Carmichael, 2008). Global consensus at the policy level on mitigating strategies of this short-lived climate forcer does not exist (Bond, 2007), although the efficacy of $\mathrm{BC}$ in perturbing the climate system manifests itself via myriads of direct and indirect effects that extend to various spatio-temporal scales. Most significantly, the presence of $\mathrm{BC}$ in air increases the absorption optical depth of the troposphere, which gives rise to a positive radiative forcing at the tropopause and a negative forcing at the surface. It is worth pointing out that biomass burning activities contribute nearly $40 \%$ to the total $\mathrm{BC}$ global budget. Therefore, the instantaneous forcing from freshly emitted $\mathrm{BC}$ is certainly non-negligible and will vary considerably seasonally

Published by Copernicus Publications on behalf of the European Geosciences Union. 
and locally. Further, deposition on snow and ice reduces the surface albedo, (Hansen and Nazarenko, 2004), thus increasing the absorption of solar radiation at the surface in polar regions. Also, an increase in $\mathrm{BC}$ concentrations could potentially impact the hydrological cycle over the south Asian monsoon region by suppressing land-sea thermal contrast via its cooling effect on the surface (Ramanathan et al., 2001). $\mathrm{BC}$ interacts with clouds (influencing its microphysical properties) in a complex manner that is not yet fully understood, let alone quantified with sufficiently high confidence (Koch and Genio, 2010). It is, therefore, essential that we are able to represent BC and its complex interplay with other Earth system components realistically in global climate models so as to better understand relevant feedback processes and eventually to reduce uncertainties in the future projections of our climate.

With the advent of Earth-system modelling, considerable efforts aim at improving the description of aerosols in global and regional climate models. By coupling atmosphere-ocean general circulation models to atmospheric chemical transport and aerosol dynamic models, prescribed aerosol fields are being replaced by a more realistic dynamic description of aerosol. Despite these efforts, significant uncertainties remain, such as those related to errors in emission estimates, poorly represented vertical distributions, uncertainties in the mixing state of $\mathrm{BC}$ with other aerosols, or errors in the size distributions.

One of the most drastic approximations in modelling the radiative forcing effect of $\mathrm{BC}$ is what is known as the homogeneous sphere approximation. In order to simplify aerosol optics computations, it is common to approximate aerosols as homogeneous spheres, which allows one to use simple Lorenz-Mie theory for computing the optical cross sections, phase function, and other optical properties. However, the morphology of dry black carbon aerosols is more realistically represented by fractal aggregates described by the scaling relation

$N_{\mathrm{s}}=k_{0}\left(\frac{R_{\mathrm{g}}}{a}\right)^{D_{\mathrm{f}}}$.

Here $N_{\mathrm{s}}$ denotes the number of spherical monomers of radius $a$ in the aggregate, $D_{\mathrm{f}}$ is the fractal dimension, $k_{0}$ denotes the fractal prefactor, and

$$
R_{\mathrm{g}}=\sqrt{\frac{1}{N_{\mathrm{s}}} \sum_{n=1}^{N_{\mathrm{s}}} r_{n}^{2}}
$$

is the radius of gyration of the aggregate (where $r_{n}$ denotes the distance of the $n$th monomer from the centre of mass). The optical properties of black carbon aggregates can be highly sensitive to their morphological characteristics. There is mounting evidence that neglecting aerosol morphology can be one of the dominant sources of error in estimating the radiative impact of black carbon (e.g. Jacobson (2001); Kahnert (2010c)).
However, accounting for morphological properties in aerosol optics computations can be highly challenging. Such studies usually require the use of numerical methods based on rigorous electromagnetic scattering theory, since methods that introduce ad hoc approximations to solving Maxwell's equations are often not sufficiently accurate when applied to black carbon aggregates (e.g. Kahnert (2010b)). Another difficulty is to obtain sufficiently reliable information about the input parameters for numerical aerosol optics calculations. For instance, by performing sensitivity studies that braced a large range of fractal parameters and refractive indices, it was found that the optical cross sections and asymmetry parameter of black carbon aggregates can vary considerably (Liu et al., 2008). On the other hand, a recent review by Bond and Bergstrom (2006) concluded that both the refractive index and the fractal dimension of freshly emitted black carbon aggregates can be narrowed down to a range that is much smaller than previously assumed. For instance, Liu et al. (2008) varied the fractal dimension of soot aggregates within the range between $D_{\mathrm{f}}=1-3$. The literature review by Bond and Bergstrom (2006) concluded that the available measurements strongly support a fractal dimension of freshly emitted black carbon of about $D_{\mathrm{f}}=1.8$.

Based on the conclusions of Bond and Bergstrom (2006), extensive aerosol optics computations for black carbon that covered the spectral range from $200 \mathrm{~nm}$ up to $12.2 \mu \mathrm{m}$ have been performed with numerically exact methods for solving the electromagnetic scattering problem (Kahnert, 2010c). The aerosol optics calculations have been coupled to chemical transport and radiative transfer models, which revealed that accounting for aggregate morphology can yield a top-ofatmosphere radiative impact that is larger by a factor of 2 as compared to corresponding results obtained with the homogeneous sphere approximation (Kahnert, 2010c).

These model computations where based on using a fractal dimension of $D_{\mathrm{f}}=1.8$ as recommended by Bond and Bergstrom (2006). However, more recent measurements suggest that this value may be too low. By use of 3-D electron tomography measurements, Adachi et al. (2007) observed a fractal dimension of $D_{\mathrm{f}}=2.4$. The large difference of this value to previously reported results is attributed to the use of 2D measurement techniques in earlier studies, which are less reliable in retrieving fractal parameters. The study by Liu et al. (2008) suggests that aggregates with a fractal dimension as high as $D_{\mathrm{f}}=2.4$ will have very different optical properties than aggregates with $D_{\mathrm{f}}=1.8$. Although the study by Liu et al. (2008) was limited to a single wavelength, it is quite possible that a change in fractal dimension from $D_{\mathrm{f}}=1.8$ to $D_{\mathrm{f}}=2.4$ can significantly alter the broadband radiative impact of black carbon. The purpose of the present study is to investigate what kind of consequences these most recent experimental results have for modelling the broadband radiative forcing effect of freshly emitted black carbon.

We perform a sensitivity analysis by investigating the direct radiative impact of $\mathrm{BC}$ aggregates with fractal 

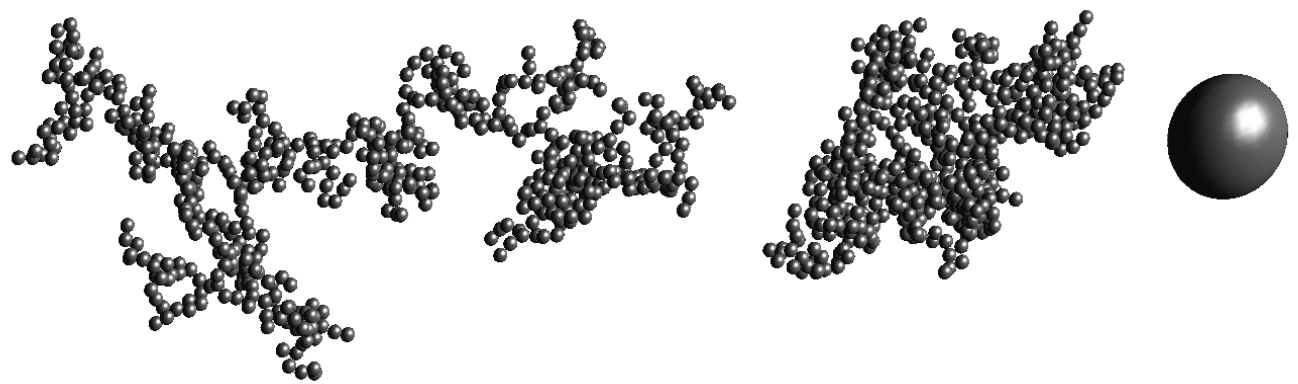

Fig. 1. Examples of model particles considered in this study: Lacy aggregates (left, $D_{\mathrm{f}}=1.8, k_{0}=1.3$ ), compact aggregates (centre, $D_{\mathrm{f}}=2.4, k_{0}=0.7$ ), volume-equivalent, homogeneous spheres (right).

dimensions $D_{\mathrm{f}}=1.8$ and $D_{\mathrm{f}}=2.4$, as well as for homogeneous $\mathrm{BC}$ spheres. We use numerically exact methods to compute optical properties of fractal aggregates in the wavelength range from $200 \mathrm{~nm}-12.2 \mu \mathrm{m}$. We emphasize that we limit this study to freshly emitted, externally mixed BC aerosols. Aged BC aggregates typically have a higher fractal dimension and, most importantly, are characterised by encapsulated geometries, i.e. they are covered by a thick coating of other aerosol compounds, such as sulphate and organics (e.g. Bond et al. (2006)). Computations are performed for the 14 wavelength bands used in the radiation scheme of the Integrated Forecasting System developed and operated at the European Centre for Medium-Range Weather Forecast. We investigate six realistic cases of aerosol distributions generated by the Multiple-scale Atmospheric Transport and Chemistry (MATCH) model over Europe. These cases are selected in such a way that they cover different surface types and different $\mathrm{BC}$ concentrations. We also investigate the sensitivity of the radiative impact to solar zenith angle. Section 2 describes the detailed methodology, while the results are presented and discussed in Sects. 3 and 4. Conclusions are given in Sect. 5

\section{Methods}

\subsection{Model geometries}

Examples of the model geometries employed in this study are shown in Fig. 1. We consider fractal aggregates with $D_{\mathrm{f}}=1.8, k_{0}=1.3$ (left, hereafter referred to as lacy aggregates), aggregates with $D_{\mathrm{f}}=2.4, k_{0}=0.7$ (centre, hereafter referred to as compact aggregates), and homogeneous, volume-equivalent spheres (right). The lacy aggregates have fractal parameters taken from the recent review by Bond and Bergstrom (2006), which are based on both observations and diffusion-limited cluster aggregation simulations (e.g. Sorensen and Roberts (1997)). The fractal parameters of the compact aggregates are taken from the more recent measurements of Adachi et al. (2007), which are based on modern 3-D electron tomography techniques (e.g. van Poppel et al. (2005)). These measurements may suggest that earlier 2D measurements tended to underestimate the fractal dimension of freshly emitted black carbon aerosols.

The monomer radius $a$ in either aggregate model is assumed to be $25 \mathrm{~nm}$. This value was found to give good agreement of modelled single-scattering albedos with measurements (Kahnert, 2010b). It is also reasonably consistent with recent field observations, which gave a median value of $a=22 \mathrm{~nm}$ (Adachi and Buseck, 2008).

In the same field observation study, aggregate sizes up to $N_{\mathrm{s}}=800$ have been observed (Adachi and Buseck, 2008). For $a=25 \mathrm{~nm}$, this would correspond to mass-equivalent aggregates with $N_{\mathrm{s}}=800 \times(22 / 25)^{3}=545$. In our calculations, we consider aggregates up to $N_{\mathrm{s}}=600$. Thus our model particles cover a size range up to volume-equivalent diameters of $420 \mathrm{~nm}$. We note that this range may be slightly larger than necessary. For instance, Kondo et al. (2011) report mass median diameters of black carbon of $150 \mathrm{~nm}$. We also note that aggregates consisting of a smaller number of monomers $N_{s}$ often are composed of primary monomers with a smaller ra$\operatorname{dius} a$. Although this would not have an effect on the absorption cross section, smaller values of $a$ may give slightly lower values of the single scattering albedo (e.g. Kahnert (2010b)). In the construction of our model particles we have neglected this effect by assuming a constant value of $a$. The model geometries are generated by use of a random cluster aggregation algorithm (Mackowski, 1994).

\subsection{Aerosol optics modelling}

To compute the optical properties of fractal black carbon aggregates, we entirely rely on numerically exact methods for solving Maxwell's equations. A recent study indicated that numerical electromagnetic scattering methods that employ ad hoc approximations based on partially or entirely neglecting electromagnetic interactions among the monomers can introduce large errors in computing optical properties of black carbon aggregates (Kahnert, 2010b). We use the superposition T-matrix code by Mackowski and Mishchenko (1996) for solving Maxwell's equations.

Our study covers the wavelength range from $200 \mathrm{~nm}-12.2 \mu \mathrm{m}$. The computations are tailored to the 
14-band shortwave radiation scheme of the Integrated Forecasting System IFS (http://www.ecmwf.int/research/ ifsdocs/CY36r1), developed at the European Centre for Medium-range Weather Forecast (ECMWF). IFS is employed as a numerical weather forecasting model, and as the atmospheric module in the Earth system climate model EC-EARTH (http://ecearth.knmi.nl). We compute black carbon optical properties for each of the 14 band mid-points. The spectral variation of the refractive index of black carbon is taken from Chang and Charalampopoulos (1990).

\subsection{Radiative transfer modelling}

Aerosol optics off-line computations can be coupled to regional (e.g. Kahnert (2010a)) and global (e.g. Aan de Brugh et al. (2011)) chemical transport models to produce 3-D fields of aerosol optical properties. Here, we use the Multiplescale Atmospheric Transport and CHemistry modelling system (MATCH) (Andersson et al., 2007) to generate a few realistic scenarios over Europe for 1-D radiative forcing studies. We use the radiative transfer modelling system libRadtran (http://www.libRadtran.org) in conjunction with the radiative transfer solver DISORT (Stamnes et al., 1988). The DISORT model is based on the discrete ordinate method (Thomas and Stamnes, 1999). The main physical approximations in DISORT are the assumption of a plane-parallel atmosphere (thus neglecting 3-D effects caused by horizontal inhomogeneity), and the use of the scalar radiative transfer equation (thus neglecting polarisation effects). The numerical accuracy is controlled by the number of "streams" (i.e. discretised polar angles) used in the calculations. In our study we use 32 streams, and we verified that the radiative transfer results have numerically converged for this choice.

\section{Results}

\subsection{Spectral optical properties of black carbon aggregates}

Figure 2 (first column) shows the optical properties of homogeneous spheres as a function of particle radius $R_{\mathrm{V}}$ and wavelength $\lambda$. The different rows show the absorption cross section $C_{\text {abs }}$ (first row), the single scattering albedo $\omega$ (second row), the asymmetry parameter $g$ (third row), and the backscattering cross section $C_{\text {bak }}$ (fourth row).

The dependency of $C_{\text {abs }}$ on $R_{\mathrm{V}}$ displays the characteristic monotonic increase. A closer analysis of the data reveals that $C_{\mathrm{abs}} \propto R_{\mathrm{V}}^{3}$ in accordance with well-known theoretical results (van de Hulst, 1957). For any fixed particle size $R_{\mathrm{V}}, C_{\mathrm{abs}}$ initially increases with wavelength $\lambda$, and then it monotonically decreases. The decrease reflects the monotonic dependency of $C_{\mathrm{abs}}$ on the size parameter $x=2 \pi R_{\mathrm{V}} / \lambda$. The initial increase in the UV and blue part of the spectrum is caused by changes in the refractive index $m$ of black carbon, which outweighs the size-parameter effect in this part of the spectrum.
This is a very important observation for the following reason. Due to the challenges involved in computing the optical properties of black carbon aggregates with electromagnetic theory, some investigators have suggested to simply use the measured value of the mass absorption cross section (MAC) at a wavelength of $550 \mathrm{~nm}$, and to extrapolate this value to other wavelengths by a $1 / \lambda$ scaling - see, e.g., Bond and Bergstrom (2006). Our results clearly show that such an approach only works over the red and IR part of the spectrum, while it completely fails in the blue and UV part of the spectrum. Thus it is essential to properly account for the spectral variation of the dielectric properties of black carbon to obtain accurate results of the spectral variation of $C_{\mathrm{abs}}$, which is crucial for obtaining reliable estimates for the shortwave radiative forcing effect of $\mathrm{BC}$.

The single scattering albedo qualitatively displays a similar dependency on size and wavelength. The asymmetry parameter $g$ mainly depends on the size parameter. It therefore reaches its highest values at large particle sizes $R_{\mathrm{V}}$ and low wavelengths $\lambda$. The backscattering cross section $C_{\text {bak }}$ of aggregates (not shown) are rather smooth functions of $\lambda$ and $R_{\mathrm{V}}$ that qualitatively behave similarly to $C_{\mathrm{abs}}$. By contrast, $C_{\mathrm{bak}}$ for homogeneous spheres displays conspicuous oscillations. It reaches its maximum values for large particle sizes at IR wavelengths.

The second column presents the differences between the optical properties of spheres and lacy aggregates. The third row shows corresponding differences between spheres and compact aggregates. Qualitatively, the second and third column look rather similar. However, Inspection of the colour bars reveals that the magnitude of the deviation between lacy aggregates and spheres is generally larger than that between compact aggregates and spheres. The most important observation is that spheres generally yield lower values of $C_{\mathrm{abs}}$ than both aggregate models, and that lacy aggregates yield larger values of $C_{\mathrm{abs}}$ than compact ones. The reason for this is that the electromagnetic field cannot penetrate deeply into a massive black carbon sphere. For this reason, much of the mass inside a spherical black carbon particle does not contribute to the absorption cross section. By contrast, in a fractal aggregate the mass is distributed such that more of the absorbing material can interact with radiation. The more lacy the aggregate, the more material interacts with the electromagnetic field, the higher $C_{\text {abs }}$ for any given volumeequivalent particle size $R_{\mathrm{V}}$. Quantitatively, the differences in $C_{\text {abs }}$ are larger between spheres and compact aggregates than between lacy and compact aggregates.

The second most important observation is that the compact sphere model predicts significantly higher values of $\omega$ than the aggregate models. Lacy aggregates yield somewhat lower values of $\omega$ than compact aggregates. The discrepancies in $C_{\text {abs }}$ and $\omega$ are very important for radiative forcing studies.

Spheres display more side- and backscattering than aggregates within a region extending from about $\left(R_{\mathrm{V}}, \lambda\right)=(50 \mathrm{~nm}$, 

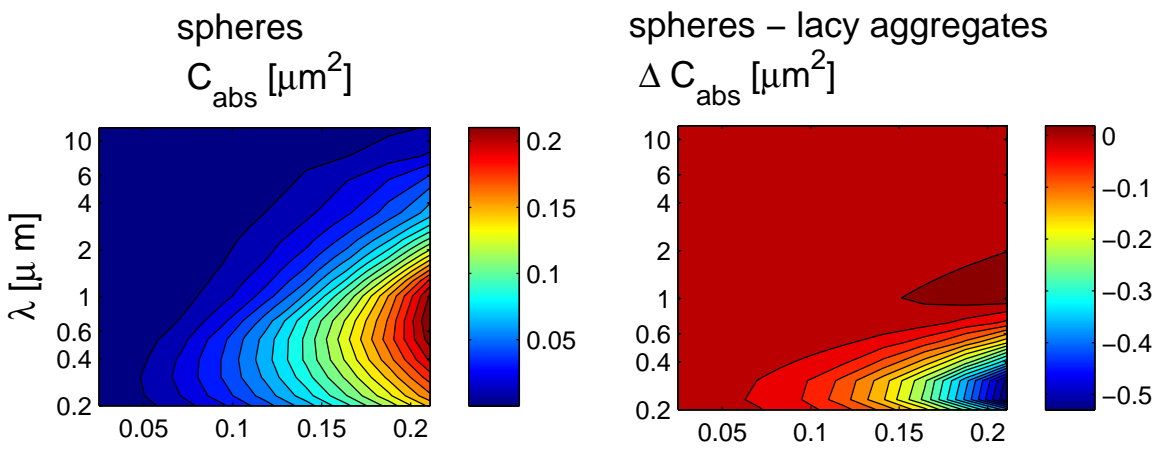

$\omega$

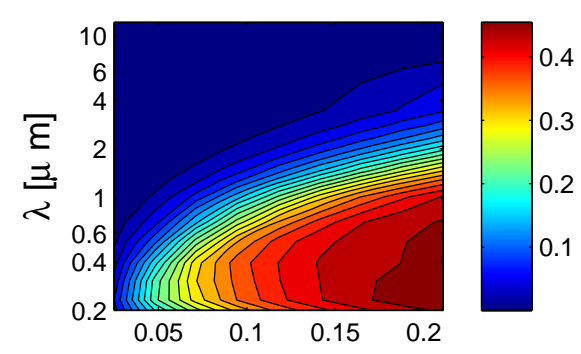

g

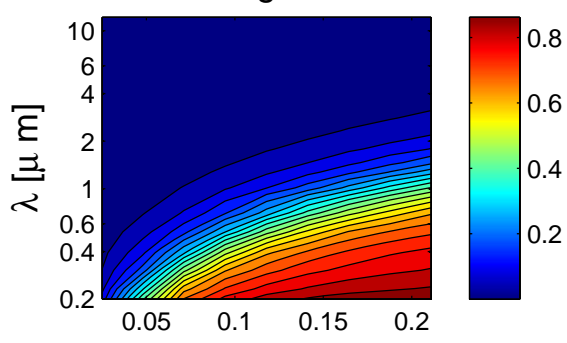

$$
\mathrm{C}_{\mathrm{bak}}\left[\mu \mathrm{m}^{2} \mathrm{sr}^{-1}\right]
$$

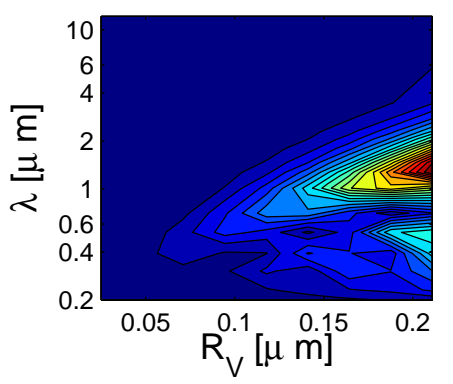

$\Delta \omega$

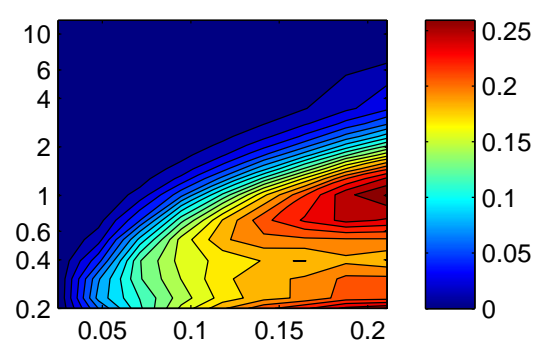

$\Delta \mathrm{g}$
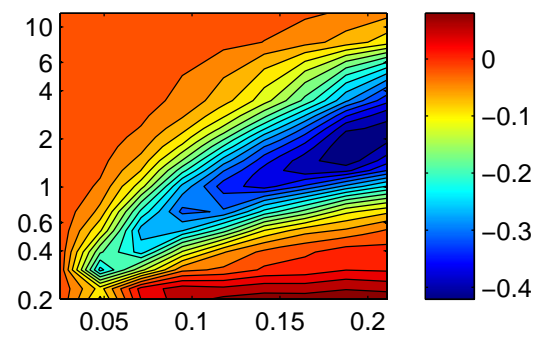

$\Delta \mathrm{C}_{\text {bak }}\left[\mu \mathrm{m}^{2} \mathrm{sr}^{-1}\right]$
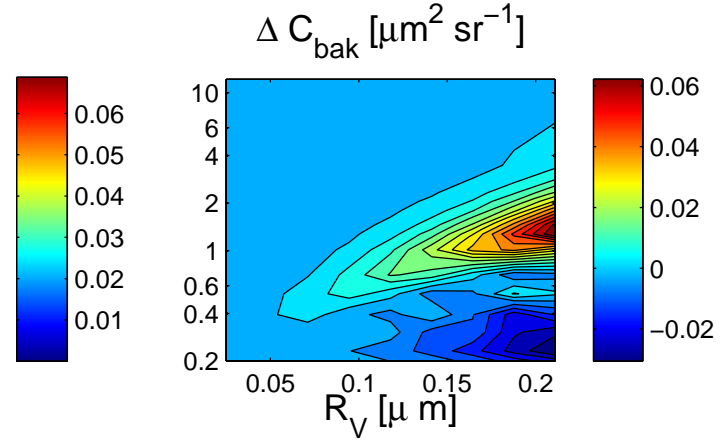
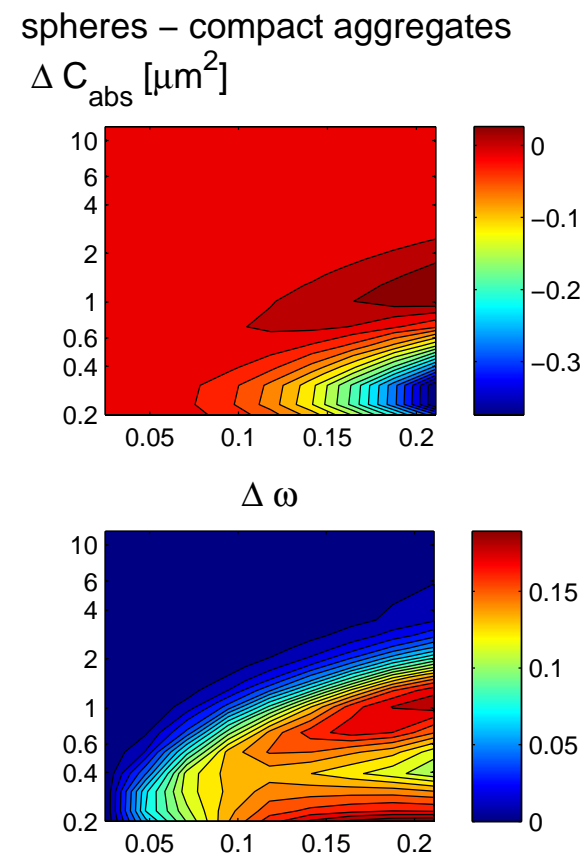

$\Delta \mathrm{g}$

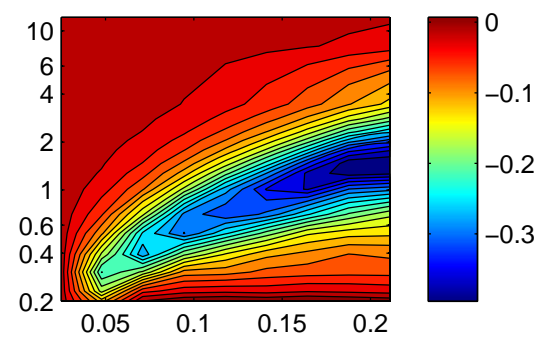

$\Delta \mathrm{C}_{\text {bak }}\left[\mu \mathrm{m}^{2} \mathrm{sr}^{-1}\right]$

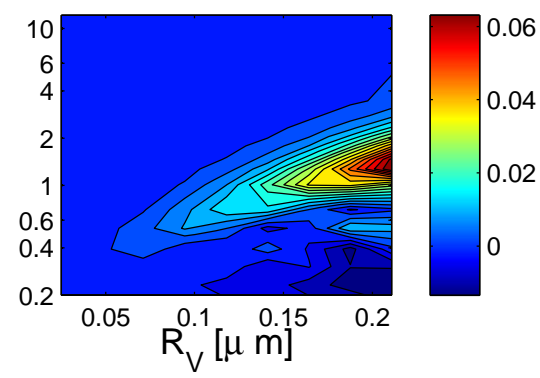

Fig. 2. Left column: optical properties of homogeneous spheres as a function of size $R_{\mathrm{V}}$ and wavelength $\lambda$. Middle column: difference in optical properties between spheres and lacy aggregates. Right column: difference in optical properties between spheres and compact aggregates. The four rows show $C_{\mathrm{abs}}, \omega, g$, and $C_{\mathrm{bak}}$, respectively.

$300 \mathrm{~nm})$ up to $(200 \mathrm{~nm}, 1.5 \mu \mathrm{m})$, which can be seen in the form of a band of minima in $\Delta g$ and maxima in $\Delta C_{\text {bak }}$.

\subsection{Comparison with measurements}

The information available from observations is considerably more scarce than the wealth of optical data we can produce with theoretical models. It is partly this scarcity of measurements that motivates the use of morphologically faithful model particles. However, the use of realistic geometries in electromagnetic scattering computations does not always guarantee to produce accurate optical properties. Difficulties in reproducing the mass absorption cross section (MAC) and the single scattering albedo of black carbon based on 
the available experimental data are discussed in Kahnert (2010b).

A recent review of available measurements (Bond and Bergstrom, 2006) concludes that a good estimate of MAC of freshly emitted, externally mixed black carbon aerosols at $\lambda=550 \mathrm{~nm}$ is $\mathrm{MAC}=7.5 \pm 1.2 \mathrm{~m}^{2} \mathrm{~g}^{-1}$. The best available measurements of the single scattering albedo suggest a value around $0.25 \pm 0.5$. Comparison of computational results for $C_{\mathrm{abs}}$ and observed values of MAC are complicated by the fact that the conversion between these two quantities relies on the mass density $\rho$, i.e.

$\mathrm{MAC}=\frac{C_{\mathrm{abs}}}{\frac{4}{3} \pi R_{\mathrm{V}}^{3} \rho}$.

For $\rho$ we can find a wide range of values in the literature. However, a critical review of the representativeness of reported data for atmospheric black carbon (Bond and Bergstrom, 2006) concludes that the most reliable estimates lie in the range $\rho=1.7-1.9 \mathrm{~g} \mathrm{~cm}^{-3}$.

At a wavelength of $\lambda=550 \mathrm{~nm}$, and assuming $\rho=1.8 \mathrm{~g} \mathrm{~cm}^{-3}, \quad$ our calculations yield $\mathrm{MAC}=5.2 \pm 0.1 \mathrm{~m}^{2} \mathrm{~g}^{-1}$ for lacy aggregates, $4.9 \pm 0.1 \mathrm{~m}^{2} \mathrm{~g}^{-1}$ for compact aggregates, and $3.3 \pm 0.4 \mathrm{~m}^{2} \mathrm{~g}^{-1}$ for spheres. The error range indicates a $95 \%$ confidence level in the least-squares fitting of $C_{\mathrm{abs}} \propto R_{\mathrm{V}}^{3}$, which is used in the computation of MAC according to Eq. (3). To obtain an overlap between the recommended value of $\mathrm{MAC}=7.5 \pm 1.2 \mathrm{~m}^{2} \mathrm{~g}^{-1}$ and the computational results, we would have to assume $\rho \lesssim 1.5 \mathrm{~g} \mathrm{~cm}^{-3}, 1.4 \mathrm{~g} \mathrm{~cm}^{-3}$, and $1.0 \mathrm{~g} \mathrm{~cm}^{-3}$ in the case of lacy aggregates, compact aggregates, and spheres, respectively. These results generally agree with those reported in Kahnert (2010b), namely, that an agreement between observed and modelled MAC values is achieved by assuming a relatively low value of the mass density $\rho$. For the compact aggregates we need to assume even lower values of $\rho$ than for lacy aggregates. For spheres we only obtain an agreement between computed and observed values of MAC by assuming a value of $\rho$ that clearly lies outside the range of reasonable mass densities. In fact, it was one of the main conclusions of the recent review by Bond and Bergstrom (2006) to abandon the use of the value of $\rho=1.0 \mathrm{~g} \mathrm{~cm}^{-3}$, which still can be found in some older studies on black carbon.

The single scattering albedo we obtain at $\lambda=550 \mathrm{~nm}$ for the larger aggregates lies at $\omega=0.25$ for lacy aggregates, 0.32 for compact aggregates, and 0.46 for spheres. Again, we see that the results obtained for the lacy aggregates seem to yield a better agreement with observations than the compact aggregates, while the results obtained for spheres are completely off the mark.

\subsection{Short-wave radiative impact of black carbon aggregates}

To generate a few realistic scenarios for radiative transfer studies, we couple the single-scattering optical properties to the regional chemical transport model MATCH. The aerosol scheme in MATCH comprises sulphate, nitrate, and ammonium aerosols as well as black carbon, primary organic carbon, mineral dust, and sea salt in four different size bins. The aerosol optical properties of all aerosols other than black carbon are currently computed by use of Mie theory, thus invoking the homogeneous sphere approximation. Details of the chemistry-optics coupling can be found in Kahnert (2009, 2010c).

As an example, Fig. 3 shows black carbon mixing ratios computed for 25 July 2006, 18:00 UTC in the lowest model layer (closest to the surface). Results are shown for ultra-fine particles $\left(R_{\mathrm{V}}<50 \mathrm{~nm}\right.$, upper left), accumulation mode particles $\left(50 \leq R_{\mathrm{V}}<500 \mathrm{~nm}\right.$, upper right), and coarse mode particles $\left(R_{\mathrm{V}}>500 \mathrm{~nm}\right.$, lower left). The lower right panel displays the single scattering albedo at $\lambda=533 \mathrm{~nm}$ in the lowest model layer. Note that the optical properties computed in MATCH involve an ensemble average over all size bins and aerosol chemical compounds. Although black carbon usually makes a small contribution to the total aerosol mass density (typically on the order of $\sim 5 \%$ ), it clearly has a strong impact on the single scattering albedo. Low values of $\omega$ often correlate with high values of black carbon mixing ratios, especially in the accumulation mode. This becomes particularly apparent when focusing in Fig. 3 on some urban emission hot spots, such as Stockholm, Oslo, Madrid, or London, which are more remote from extensive continental area sources.

We pick six geographic locations (indicated by circles in Fig. 3) for which we conduct detailed radiative transfer studies. Among those, we have chosen two continental sites (one North and one South of the Alps) which represent more polluted conditions, one site in Scandinavia, which represents a situation with both long-range transported air pollution and local emissions, and one at the West coast of Ireland, which is dominated by marine aerosols. We also pick two locations over water, one over the North Sea with rather clean air, and one over the Mediterranean Sea with more polluted air.

Figure 4 shows vertical profiles of the spectral extinction at $\lambda=533 \mathrm{~nm}$ for the six different locations. The three curves in each panel are based on using lacy aggregates (red), compact aggregates (blue), and spheres (green) for computing the optical properties of black carbon. Despite the fact that the three models yield rather large differences for the absorption cross section (see Fig. 2), the extinction profiles are very similar. This is expected, as the extinction is dominated by the contributions from scattering aerosols, which are computed by the same method in all three cases. The site in Northern Italy $\left(45^{\circ} \mathrm{N} 12^{\circ} \mathrm{E}\right.$, upper left) is strongly dominated by local anthropogenic sources. This is reflected by a sharp increase in the spectral extinction near the surface. The site South-east 

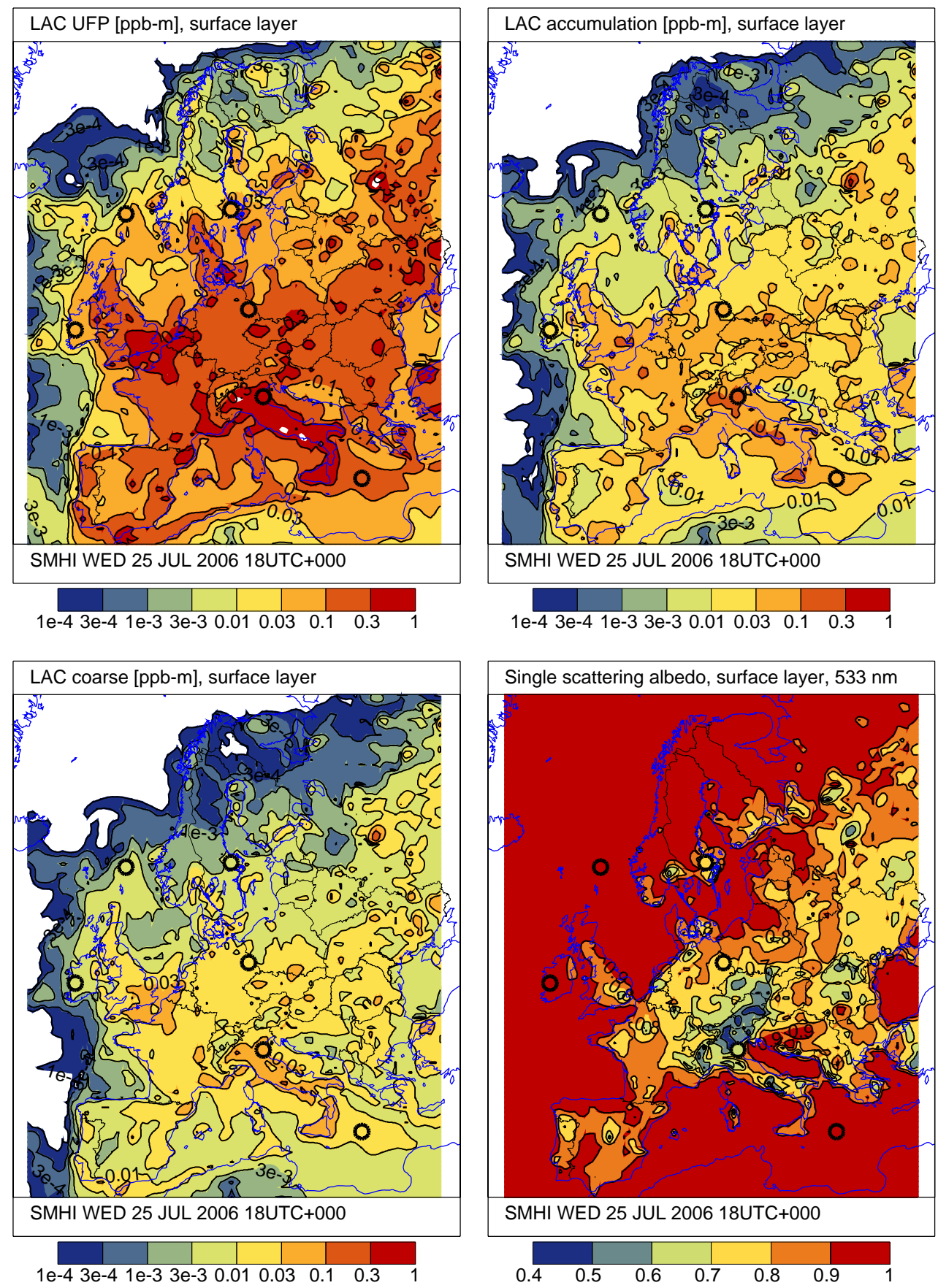

Fig. 3. Black carbon mass mixing rations $(\mu \mathrm{g} / \mathrm{kg}$ ) for size bins with diameters $<100 \mathrm{~nm}$ (upper left), $100 \mathrm{~nm}-1 \mu \mathrm{m}$ (upper right), and $>1 \mu \mathrm{m}$ (lower left), as well as single scattering albedo $\omega$ (lower right) at $\lambda=533 \mathrm{~nm}$. The maps show results for 25 July 2006 18:00 UTC in the model layer closest to the surface. Also indicated are six locations chosen for radiative transfer case studies.

of Berlin $\left(52^{\circ} \mathrm{N} 14^{\circ} \mathrm{E}\right.$, upper right) displays a similar peak near the ground. In addition, there is a pronounced maximum at around $1.3 \mathrm{~km}$, and a smaller peak at $2.2 \mathrm{~km}$, which indicate contributions from long-range transported aerosols. This becomes even more pronounced at the site near Uppsala $\left(60^{\circ} \mathrm{N} 17^{\circ} \mathrm{E}\right.$, centre left), where the long-range peak near
$2 \mathrm{~km}$ is actually larger than the surface contribution. South of Galway $\left(53^{\circ} \mathrm{N} 9^{\circ} \mathrm{W}\right.$, centre right) we see much less contributions from local sources, but a pronounced peak within the boundary layer, which mainly originates from nearby sea salt emissions. Over the North Sea $\left(62^{\circ} \mathrm{N} 0^{\circ}\right.$, lower left) there is a large peak in the surface layer caused by strong 
$45^{\circ} \mathrm{N} 12^{\circ} \mathrm{E}$

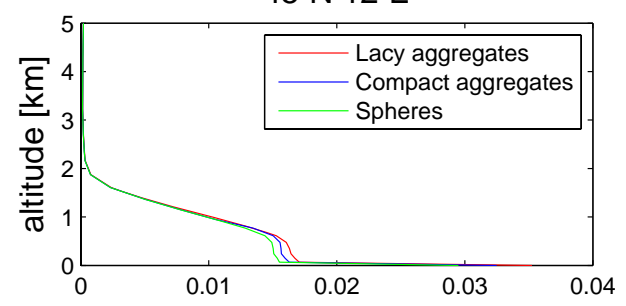

$60^{\circ} \mathrm{N} 17^{\circ} \mathrm{E}$

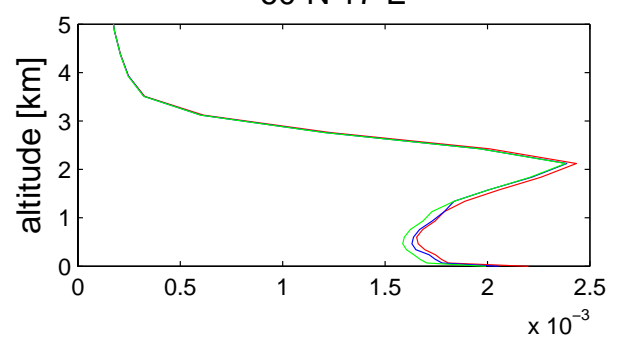

$62^{\circ} \mathrm{N} 0^{\circ}$

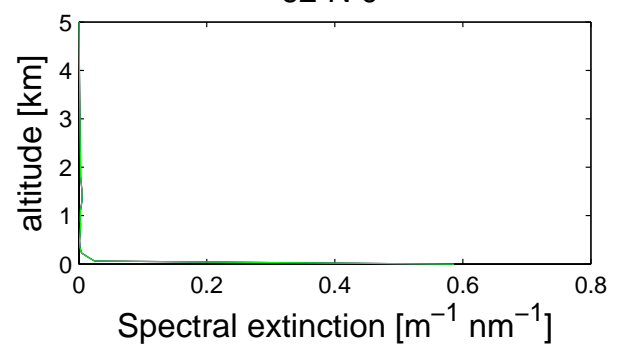

$52^{\circ} \mathrm{N} 14^{\circ} \mathrm{E}$

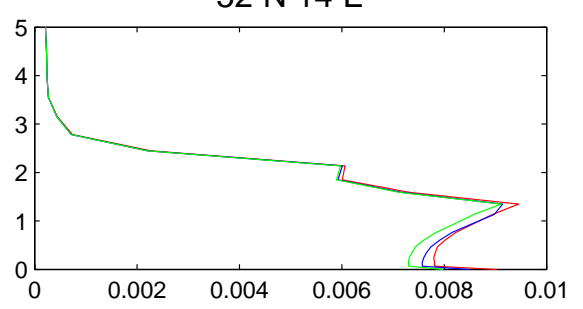

$53^{\circ} \mathrm{N} 9{ }^{\circ} \mathrm{W}$

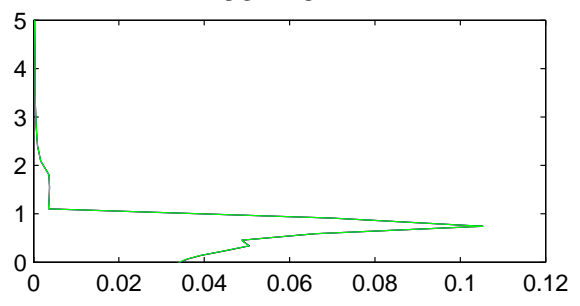

$36^{\circ} \mathrm{N} 18^{\circ} \mathrm{E}$

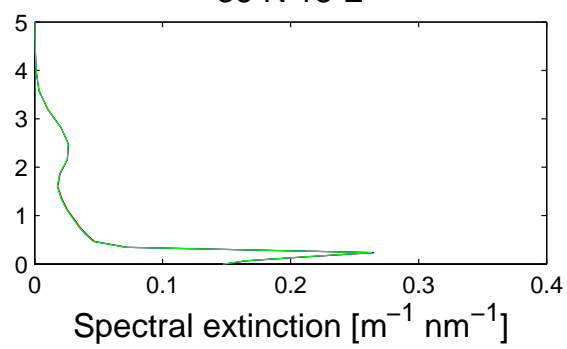

Fig. 4. Vertical profiles of the spectral extinction at $\lambda=532 \mathrm{~nm}$ for six different locations as indicated in the figure headings. Results are based on the lacy aggregate (red), compact aggregate (blue), and homogeneous sphere models (green).

local sea salt emissions. By contrast, the Mediterranean site $\left(36^{\circ} \mathrm{N} 18^{\circ} \mathrm{E}\right.$, lower right) displays higher spectral extinction a bit higher up in the boundary layer, as well as another broad maximum between $2-3 \mathrm{~km}$, which is caused by long-range transported aerosols.

Figure 5 shows corresponding profiles of the single scattering albedo. As expected, we observe much stronger variations among the three different models for black carbon optics than for the spectral extinction. In general, the lacy aggregate model gives the lowest, while the sphere model gives the highest values of $\omega$. This agrees with our expectations based on the single-scattering results shown in Fig. 2. The sites in Italy (upper left), Germany (upper right), and Sweden (centre left) display a steady decrease towards the surface, which is caused by elevated black carbon concentrations due to local emissions. Qualitatively, a similar decrease in $\omega$ is seen at the Irish site (centre right); but quantitatively, this decrease is rather small. Over the North Sea (lower left), $\omega$ is almost unity near the surface, thus confirming the dominance of non-absorbing sea salt. The local minima higher up in the boundary layer as well as around $2 \mathrm{~km}$ altitude indicate the presence of somewhat higher contributions from long-range transported black carbon. The Mediterranean location (lower right) displays two local minima around $1 \mathrm{~km}$ and $4.5 \mathrm{~km}$.

Figure 6 presents profiles of the asymmetry parameter $g$. The most noteworthy observation is that the order of the three models is now changed. Compact aggregates yielded results for the extinction and single scattering albedo that lied in between those computed for spheres and lacy aggregates. By contrast, the compact aggregate model gives the highest values of $g$ among the three different models. A closer analysis of the single-scattering optical properties of black carbon shows that for larger particles in the visible part of the spectrum, the asymmetry parameter of black carbon computed with the compact aggregate model can be larger than that computed with the lacy aggregate model by as much as 0.1 . This will soon become important when we try to understand the radiative impact computed with the aggregate models.

The vertical profiles of spectral extinction, $\omega$, and $g$ are used as input to radiative transfer computations. All computations are performed for a cloud-free, plane parallel atmosphere. Molecular extinction is accounted for by assuming a standard mid-latitude atmosphere. The spectral surface albedo is taken from the Moderate Resolution Imaging 


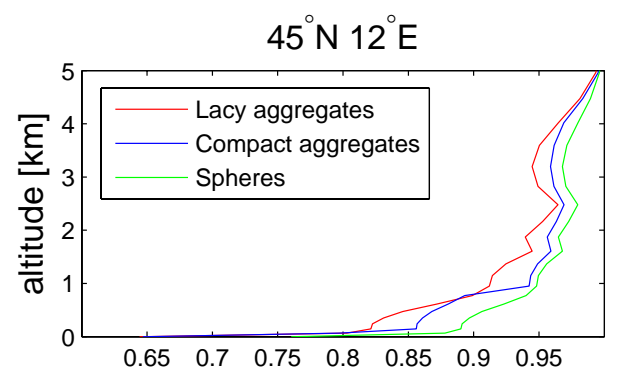

$60^{\circ} \mathrm{N} 17^{\circ} \mathrm{E}$

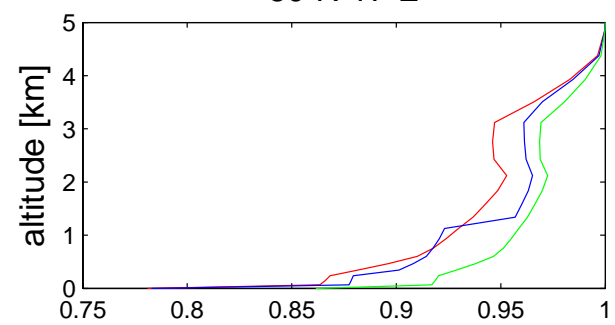

$62^{\circ} \mathrm{N} 0^{\circ}$

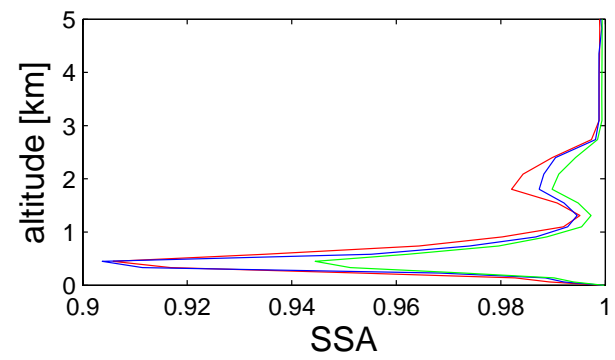

Fig. 5. As Fig. 4, but for the single scattering albedo.

Spectroradiometer (MODIS) Statistical Product that has 16day periodicity and provides albedo values at $1 \times 1$ degree resolution at 7 spectral and 3 broadband intervals $(0.47 \mu \mathrm{m}$, $0.555 \mu \mathrm{m}, 0.659 \mu \mathrm{m}, 0.858 \mu \mathrm{m}, 1.24 \mu \mathrm{m}, 1.64 \mu \mathrm{m}, 2.1 \mu \mathrm{m}$, and broadband $0.3-0.7 \mu \mathrm{m}, 0.3-5.0 \mu \mathrm{m}, 0.7-5.0 \mu \mathrm{m})$. The white-sky albedo values from year 2004 are used. The computed spectral radiative net fluxes are integrated over the solar spectrum to obtain the broadband net flux $F$. These computations are repeated with the three different models used for black carbon, as well as for an atmosphere free of black carbon. The radiative impact is defined as the difference between the net fluxes computed with and without black carbon, i.e.

$\Delta F=F($ with $\mathrm{BC})-F($ without $\mathrm{BC})$

Figure 7 presents results computed for 25 July at 13:00 UTC. Depending on geographic location, this corresponds to a solar zenith angle (SZA) in the range $32^{\circ}-46^{\circ}$, see Table 1 for details. At all six locations, we clearly see that the simple homogeneous sphere model (green) underestimates the radiative impact at the top of atmosphere (TOA) as compared to the more realistic aggregate models. At the $52^{\circ} \mathrm{N} 14^{\circ} \mathrm{E}$

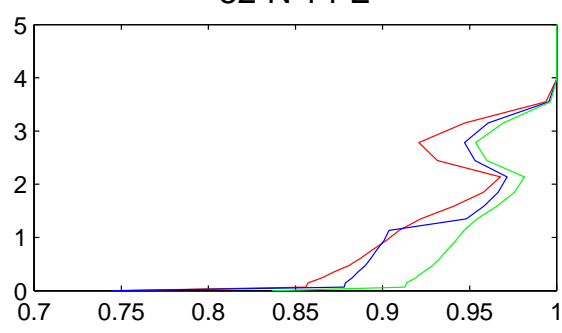

$53^{\circ} \mathrm{N} 9^{\circ} \mathrm{W}$

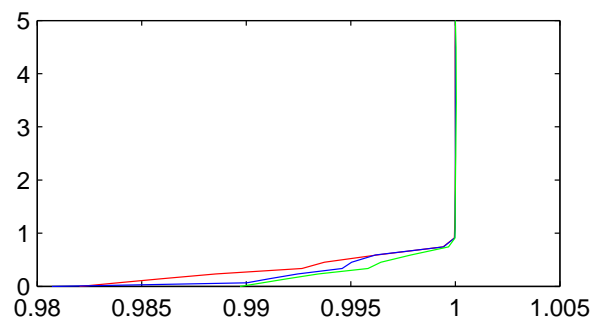

$36^{\circ} \mathrm{N} 18^{\circ} \mathrm{E}$

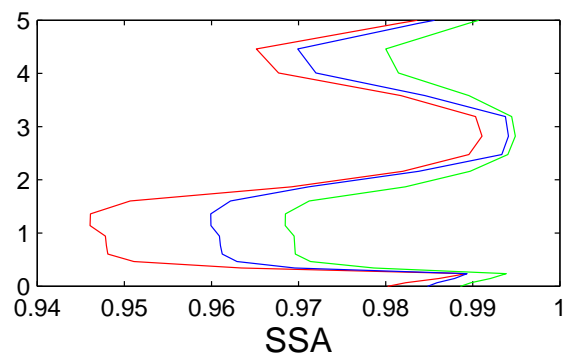

Table 1. Ratios of SW radiative impacts $\delta F$ (as explained in the text) at TOA and BOA at six different locations. Also shown are the solar zenith angles (SZA) at 13:00 UTC.

\begin{tabular}{lllllllll}
\hline & & \multicolumn{3}{c}{ TOA } & \multicolumn{3}{c}{ BOA } \\
Lat & Lon & SZA & $\delta F_{1}$ & $\delta F_{2}$ & $\delta F_{3}$ & $\delta F_{1}$ & $\delta F_{2}$ & $\delta F_{3}$ \\
\hline $45^{\circ} \mathrm{N}$ & $12^{\circ} \mathrm{E}$ & $33^{\circ}$ & 1.73 & 1.62 & 1.07 & 1.79 & 1.55 & 1.15 \\
$52^{\circ} \mathrm{N}$ & $14^{\circ} \mathrm{E}$ & $39^{\circ}$ & 1.76 & 1.61 & 1.09 & 1.80 & 1.57 & 1.15 \\
$60^{\circ} \mathrm{N}$ & $17^{\circ} \mathrm{E}$ & $46^{\circ}$ & 2.02 & 1.66 & 1.22 & 1.87 & 1.87 & 1.00 \\
$53^{\circ} \mathrm{N}$ & $9^{\circ} \mathrm{W}$ & $33^{\circ}$ & 1.93 & 1.35 & 1.43 & 1.83 & 1.42 & 1.29 \\
$62^{\circ} \mathrm{N}$ & $0^{\circ}$ & $43^{\circ}$ & 2.09 & 1.34 & 1.56 & 1.75 & 1.42 & 1.23 \\
$36^{\circ} \mathrm{N}$ & $18^{\circ} \mathrm{E}$ & $32^{\circ}$ & 1.71 & 1.47 & 1.16 & 1.75 & 1.37 & 1.28 \\
\hline
\end{tabular}

bottom of atmosphere (BOA) the sphere model yields too little negative forcing. These observations are consistent for all six locations. At the more polluted sites the difference between the two aggregate models is rather small, while at the more remote sites the radiative impact of compact aggregates lies halfway between that of spheres and that of lacy aggregates. 

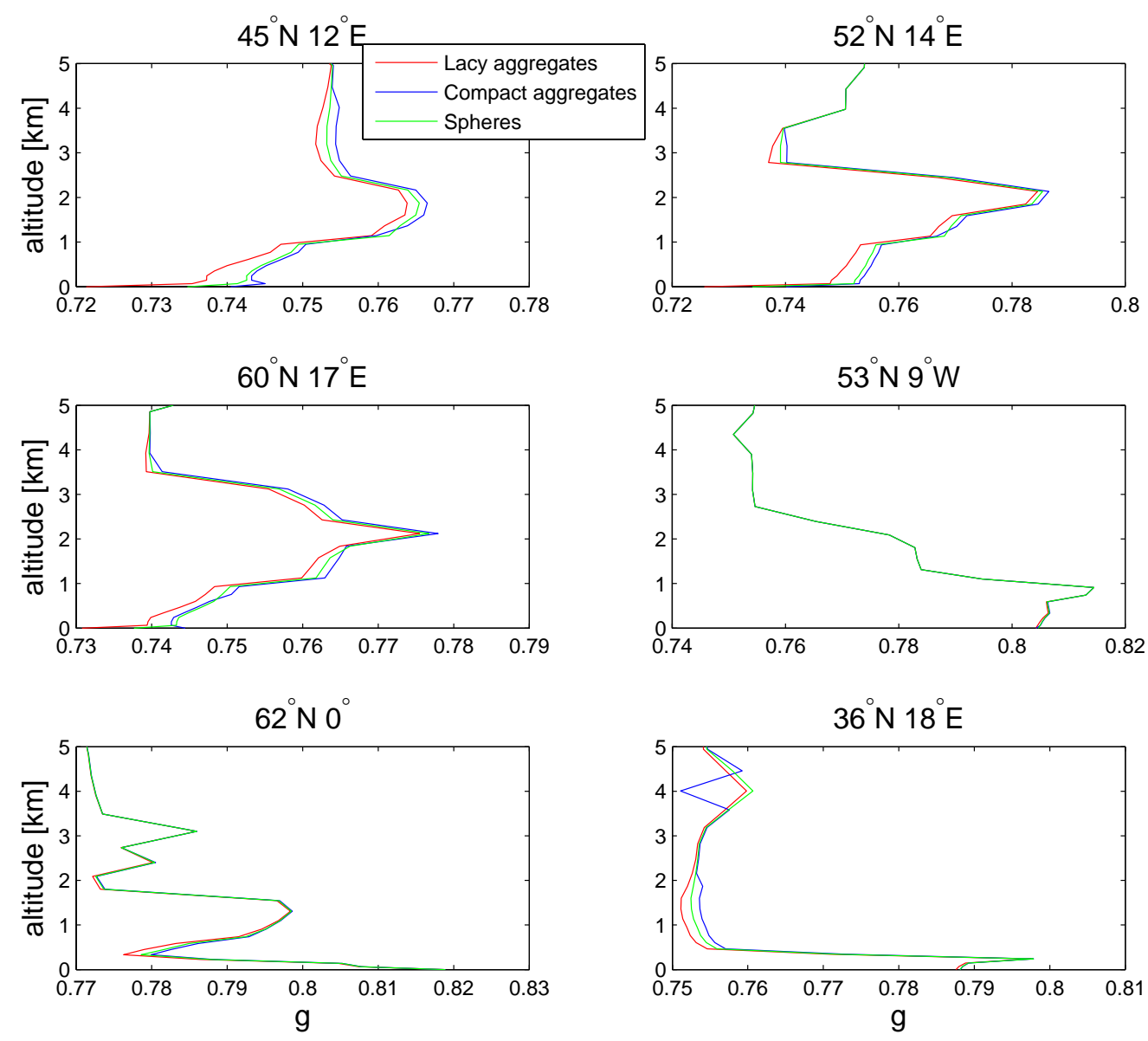

Fig. 6. As Fig. 4, but for the asymmetry parameter.

Table 1 shows a quantitative comparison of the radiative impacts at TOA an BOA obtained with the three aggregate models. The table shows the quantities

$\delta F_{1}=\Delta F$ (lacy aggregates) $/ \Delta F$ (spheres)

$\delta F_{2}=\Delta F$ (compact aggregates) $/ \Delta F$ (spheres)

$\delta F_{3}=\Delta F$ (lacy aggregates) $/ \Delta F$ (compact aggregates)

At TOA lacy aggregates yield a radiative impact that is larger than that computed with the sphere model by a factor of 1.7-2.1. The compact aggregate model gives a radiative impact that exceeds that of spheres by a factor of 1.3-1.7. The radiative impact computed with the lacy aggregate model is higher than that obtained with the compact aggregate model by a factor of 1.1-1.6\%. Table 2 shows a similar comparison for only the Italian site but for different values of SZA. Although the radiative impact $\Delta F$ strongly depends on SZA, the ratios $\Delta F$ (model1) $/ \Delta F$ (model2) vary only little with SZA.

In general, the tables clearly show that the errors introduced by the homogeneous sphere model are substantial. There can be little doubt that neglecting the fractal morphology of BC in radiative impact studies will result in large er-
Table 2. $\delta F$ as in Table 1 at TOA and BOA at a location $45^{\circ} \mathrm{N}$ $12^{\circ} \mathrm{E}$ for three different SZA.

\begin{tabular}{ccccccc}
\hline & \multicolumn{3}{c}{ TOA } & \multicolumn{3}{c}{ BOA } \\
SZA & $\delta F_{1}$ & $\delta F_{2}$ & $\delta F_{3}$ & $\delta F_{1}$ & $\delta F_{2}$ & $\delta F_{3}$ \\
\hline $22^{\circ}$ & 1.75 & 1.61 & 1.09 & 1.79 & 1.55 & 1.15 \\
$40^{\circ}$ & 1.72 & 1.62 & 1.06 & 1.76 & 1.54 & 1.14 \\
$60^{\circ}$ & 1.77 & 1.68 & 1.05 & 1.76 & 1.54 & 1.14 \\
\hline
\end{tabular}

rors. However, the differences caused by different assumptions about the fractal parameters are smaller than those between the spherical model and each of the aggregate models. Although the maximum ratios $\delta F_{3}$ of the two fractal models are rather large, we also see that they occur at the cleanest locations (over the North Sea and at the Irish site), where the magnitude of the radiative impact $\Delta F$ is smallest (cf. Fig. 7). Over the more polluted sites, where the radiative impact is highest, the ratio $\delta F_{3}$ is on the order of 1.1-1.2 at TOA, and $1.0-1.3$ at BOA. 

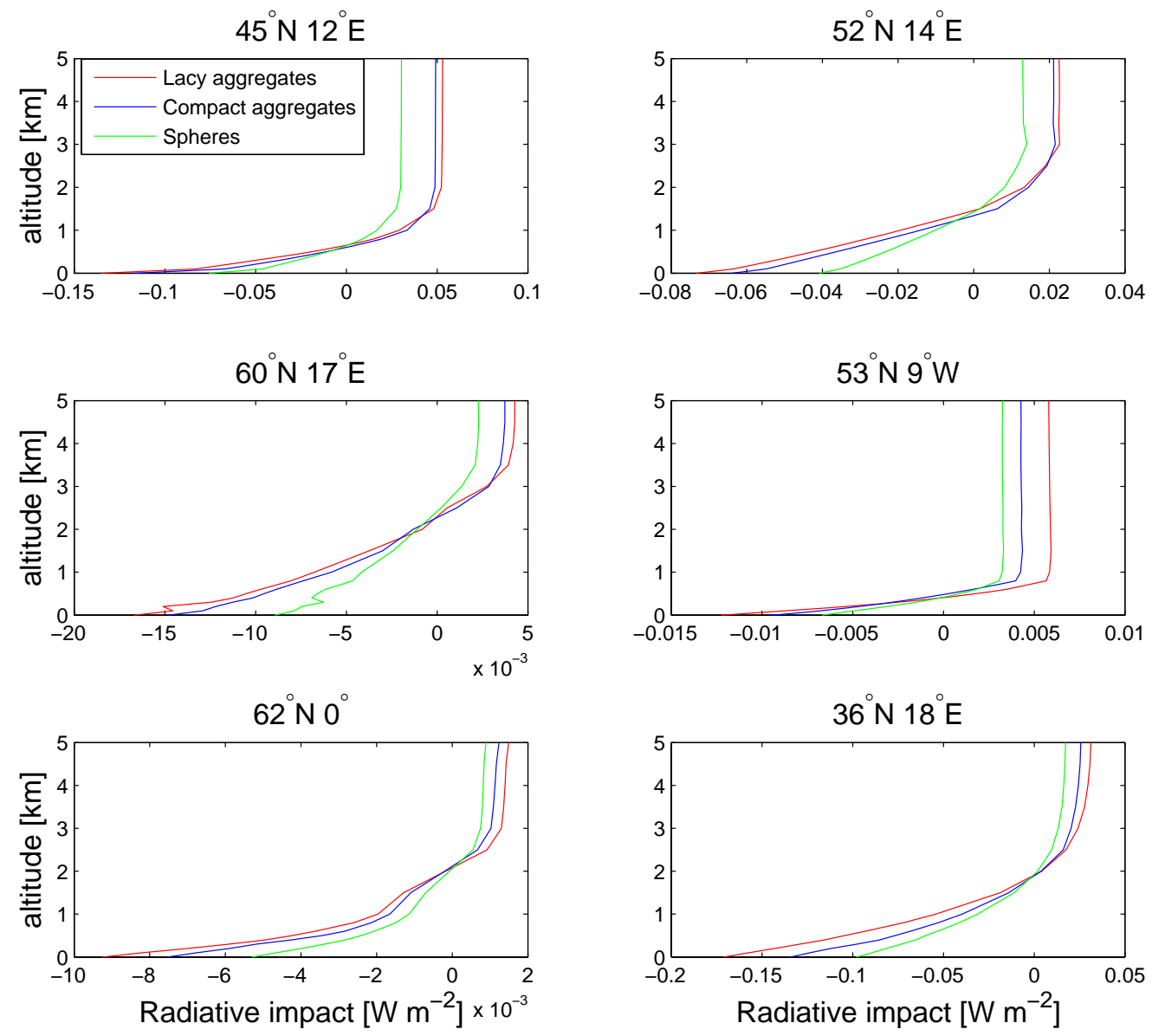

Fig. 7. As Fig. 4, but for the shortwave radiative impact (integrated over all SW wavelengths).

A plausible explanation for these observations can be found by inspecting the single scattering optical properties. Briefly stated, we have, over large parts of the size range and wavelength spectrum

$$
\begin{aligned}
C_{\text {abs }} \text { (spheres) } & \left.<C_{\text {abs }} \text { (lacy aggregates }\right) \\
\omega \text { (spheres }) & >\omega \text { (lacy aggregates }) \\
g \text { (spheres }) & <g \text { (lacy aggregates }) .
\end{aligned}
$$

So, spheres yield less absorption, more scattering in relation to absorption, and more isotropic scattering, hence more side- and backscattering. All three effects are additive in radiative net flux computations, resulting in a lower positive TOA radiative impact for spheres. On the other hand, we have (over a large part of the size range, and at least over the visible part of the spectrum, where the solar spectral radiance is highest)

$$
\begin{aligned}
C_{\mathrm{abs}} \text { (compact aggregates) } & <C_{\mathrm{abs}} \text { (lacy aggregates) } \\
\omega \text { (compact aggregates }) & >\omega \text { (lacy aggregates) } \\
g \text { (compact aggregates }) & >g \text { (lacy aggregates). }
\end{aligned}
$$

So, compact aggregates yield less absorption and more scattering in relation to absorption. Again, these two effects are additive and give a larger diffuse upwelling flux, thus a lower positive radiative impact at TOA for compact aggregates. The intriguing difference to the comparison with spheres is that the asymmetry parameter of compact aggregates is generally larger than that of lacy aggregates, which gives less side- and backscattering, thus less radiative cooling to counter-act the warming effect of absorption at TOA. The asymmetry parameter enters into the radiative transfer equation through the parameterisation of the HenyeyGreenstein phase function (Henyey and Greenstein, 1941). So in case of the two aggregate models, the asymmetry parameter can counter-act the effect of $C_{\text {abs }}$ and $\omega$, thus moving the radiative impact profiles of lacy and compact aggregates in Fig. 7 closer together. However, in clean air the black carbon concentration, and thus its contribution to the optical depth is so low that even single-scattering events by $\mathrm{BC}$ aerosols are very rare, and the radiative forcing effect of $\mathrm{BC}$ is completely dominated by $\mathrm{BC}$ absorption. Under more polluted conditions, the radiative impact is at least to some extent influenced by single-scattering events, in which case the compensating effect of the error in the asymmetry parameter brings the radiative forcing results for lacy and 
compact aggregates closer together. This may explain why the differences between the radiative impacts of lacy and compact aggregates are smallest at the more polluted sites. However, we note that under extremely polluted conditions multiple scattering effects may become important. With each order of scattering the form of the phase function, thus the asymmetry parameter becomes less important. So, multiple scattering would reduce the impact of differences in asymmetry parameters.

These considerations make it clear that the relatively good agreement between the two aggregate models under polluted conditions are caused by a rather fortuitous error cancellation, rather than by a close agreement of the single-scattering optical properties. As we saw, this error cancellation depends on the chemical state of the atmosphere. It may also depend on other conditions, such as surface albedo, underlying clouds, vertical distributions of aerosols, etc. We therefore conclude that model calculations that use an aggregate model with non-representative fractal parameters $D_{f}$ and $k_{0}$ will, in general, yield incorrect estimates of the radiative forcing effect of black carbon, even though under favourable conditions there may occur some lucky error cancellations due to the opposing effects of different error sources.

\section{Discussion}

We studied the significance of morphological properties for the radiative impact of black carbon aerosols. The general idea was to employ morphologically faithful model particles and numerically exact electromagnetic scattering and radiative transfer models. By numerically exact models we mean numerical methods of which the accuracy is only limited by numerical aspects, rather than by inaccuracies caused by approximations in the description of the relevant physics. The radiative impacts under different conditions were studied for two different assumptions about the fractal aggregate structure of the aerosols, and the results were compared to computations based on the frequently employed homogeneous sphere approximation. With this approach we can gauge the importance of aerosol optics for climate forcing studies, and understand the complex interplay of microphysical aerosol morphology, single-particle optical properties, and macroscopic radiative transfer processes.

Our study of the optical properties of single particles revealed that the homogeneous sphere model generally predicts the lowest values of the absorption cross section $C_{\mathrm{abs}}$, while the lacy aggregate model predicts the highest values. The physical reason is that the electromagnetic energy is quickly attenuated inside a massive, highly absorbing black carbon sphere. As a result, much of the mass residing inside the sphere does not interact with the radiation, and therefore does not contribute to the absorption. Even in a compact aggregate, some of the mass near the centre of the aggregate is partially screened by the surrounding monomers. By con- trast, in a lacy aggregate the material is distributed such that most of the mass can interact with the electromagnetic field.

We further found that spheres predict significantly larger values of the single scattering albedo $\omega$ than the aggregate models. These differences in single-scattering optical properties result in significant differences in radiative impacts. The homogeneous sphere model under-predicts the TOA radiative impact as compared to the more realistic aggregate models. For lacy aggregates we computed TOA radiative impacts that exceeded those obtained with the sphere model by more than a factor of 2 . This is consistent with results reported in Kahnert (2010c). The errors introduced by the homogeneous sphere approximation are caused by the combined effect of underestimating absorption and overestimating the relative importance of scattering. We used our chemical transport model MATCH to generate six case studies, which were meant to represent different conditions in terms of local emission sources, long-range transport of air pollution, and different surface albedos. In all cases we consistently found a significant bias of the radiative impact predicted by the homogeneous sphere model.

Differences in radiative impacts computed by the lacy and compact aggregate models were generally smaller than those between spheres and aggregates. This was especially the case under more polluted conditions, under which the radiative impact of black carbon is largest. The differences in asymmetry parameters $g$ among the different models may play an essential role in explaining these results. Spheres generally yield smaller values of $g$ than each of the two aggregate models. Consequently, spheres predict more side- and backscattering, which reduces the radiative warming effect at TOA. The small values of $C_{\mathrm{abs}}$ and the high values of $\omega$ obtained for spheres have exactly the same effect, resulting in a smaller TOA radiative impact as compared to the aggregate models in all six case studies. By contrast, analysis of the optical properties of lacy and compact aggregates reveals that there are two competing effects. On the one hand, compact aggregates give lower values of $C_{\mathrm{abs}}$ and higher values of $\omega$ than lacy aggregates, which results in a lower positive TOA radiative impact. On the other hand, for visible wavelengths compact aggregates give higher values of $g$ than lacy aggregates, which results in less radiative cooling, thus more radiative warming at TOA. To what extent this can partially cancel the effects of $C_{\mathrm{abs}}$ and $\omega$ strongly depends on atmospheric conditions.

Error cancellation is a highly unpredictable and unreliable mechanism for producing accurate modelling results. In particular, differences in radiative impact between lacy and compact aggregates can also depend on other conditions not investigated in this study. This suggests that obtaining reliable information on the fractal parameters of black carbon aerosols is an important prerequisite for reliable modelling estimates of the radiative impact of black carbon.

There are still important open questions that limit the scope of conclusions we can draw from our results. Most 
importantly, there are unresolved issues in the comparison of computed and measured mass absorption cross sections (MAC) of black carbon. We noted a clear discrepancy between MAC results obtained with the homogeneous sphere model and the range of observations reported in Bond and Bergstrom (2006). However, we also noted some difficulties in reproducing the measurements with the aggregate models. Although the aggregate models give much more reasonable MAC values than the homogeneous sphere model, they still seem to be too low in comparison to the measurements. Also, the presumably more realistic compact aggregate model gave less agreement with the observed MAC values than the lacy aggregate model. There are different possible explanations for these discrepancies.

1. A potentially important source of uncertainty is the refractive index of black carbon (Kahnert, 2010b). An increase in MAC could be achieved by assuming a higher value of $\operatorname{Im}(m)$. However, this would be difficult to reconcile with the available measurements of $m$. Our calculations rely on refractive index values reported by Chang and Charalampopoulos (1990), which are among the highest values of $\operatorname{Im}(m)$ within the range recommended by Bond and Bergstrom (2006). Also, as pointed out by Bond and Bergstrom (2006), the variation in $m$ among different measurements of black carbon is most plausibly explained by varying amounts of void fractions within the carbonaceous material. Physically, and increase in $\operatorname{Im}(m)$ would be caused by a decrease in the amount of void fractions. This would only be consistent with assuming a higher mass density, which, in turn, would reduce MAC and thereby counteract the effects of increasing $\operatorname{Im}(m)$.

2. Calculations and comparisons with measurements rely on a fairly precise knowledge of optical properties (MAC, $\omega)$, material properties $(m, \rho)$, and morphological properties $\left(a, D_{\mathrm{f}}, k_{0}\right)$. In our study, we largely rely on the recent review by Bond and Bergstrom (2006) (which provides a critical and comprehensive evaluation of the available experimental data) and on other recent studies (Adachi et al., 2007; Adachi and Buseck, 2008). However, many of these data originate from different sources, in which different sub-sets of black carbon properties have been measured. It is unclear to what extent various properties compiled from different studies on different samples can yield closure of morphological, material, and optical properties. It would therefore be extremely helpful to conduct more observations in which a complete characterisation of black carbon samples is achieved, i.e. studies that comprise both optical, material, and morphological properties simultaneously. Also, it would be desirable to have a more complete characterisation of the optical properties of black carbon. Although less relevant for understanding the climate forcing effect of black carbon, measurements of differential scattering behaviour and polarisation properties would be extremely helpful for further constraining aerosol optics models.

3. The discrepancies between modelled and observed MAC values may even be caused by over-simplifying assumptions in our aggregate models. This possibility may seem surprising in view of the highly complex fractal aggregate models we used (see Fig. 1). However, despite the high level of geometrical details in our model particles, there are certain aspects that we have not taken into account. We assumed that the aggregates consist of monomers that touch each other in only one point. More realistic models would account for sintered morphologies. For $\mathrm{TiO}_{2}$ aggregates, it has been shown that sintering can increase the extinction cross section, especially in the UV and visible spectral range (Wriedt et al., 2010). Perhaps even more importantly, we know that freshly emitted black carbon aggregates are usually not "naked" particles, but coated by a fine film of organic material. Such morphological features have been neglected in our computations. As we mentioned in the introduction section, it is well understood that encapsulation of aged black carbon particles in larger (typically accumulation size) liquid aerosol droplets significantly enhances their absorption properties (e.g. Bond et al. (2006)). By encapsulation we mean a coating that is so thick that the black carbon aggregate is completely or almost completely covered by the soluble material. The black carbon volume fraction of such encapsulated geometries is typically below $10 \%$, and it rarely exceeds $20 \%$. It is possible, but as yet not known, that a thin coating with organic material somewhat increases $C_{\mathrm{abs}}$ and thus MAC of freshly emitted black carbon aggregates. Both sintering and coating are morphological features that cannot be accounted for in the superposition T-matrix method we used for solving Maxwell's equations. Such studies would require the use of different numerical methods, such as the discrete dipole approximation (e.g. Draine (2000)).

\section{Conclusions}

The results of this study clearly demonstrate that the commonly used homogeneous sphere approximation, which is the basis of Lorenz-Mie theory (Mie, 1908), introduces unacceptably high biases in radiative forcing estimates of black carbon. Morphologically more realistic aggregate models give a better representation of observed mass absorption cross sections and are likely to significantly improve our estimates of the climate forcing effect of black carbon. Differences in fractal dimensions and fractal prefactors have, at first glance, a lesser impact on radiative forcing as compared to the differences between homogeneous spheres and fractal aggregates. However, a closer analysis reveals that this 
is mainly caused by error cancellations that can be highly sensitive on atmospheric conditions. To thoroughly understand the connection between aerosol morphology and radiative impact, more comprehensive measurements are needed of morphological, physical, and optical properties. Experimental studies need to be supplemented by more detailed theoretical studies of potentially important effects, such as sintering and coating.

Another important finding of our study is that the spectral variation of the absorption cross section is non-monotonic. The simple $1 / \lambda$ scaling law only holds in the red and IR part of the spectrum, but not at shorter wavelengths. Thus we strongly discourage the use of measured values of the mass absorption cross section at a single wavelength in conjunction with a simple $1 / \lambda$ extrapolation to other wavelengths; it is essential to properly account for the spectral variation of the dielectric properties of $\mathrm{BC}$ in broadband radiative forcing simulations.

Acknowledgements. The authors thank D. Mackowski for providing his program for aggregated-particle generation. D. Mackowski and M. Mishchenko are acknowledged for making their superposition T-matrix program publicly available. Y. Okada and A. Kokhanovsky are acknowledged for making their numerical orientational averaging routines publicly available. This work was supported by the Swedish Research Council under contract number 80438701 .

Edited by: N. Riemer

\section{References}

Aan de Brugh, J. M. J., Schaap, M., Vignati, E., Dentener, F., Kahnert, M., Sofiev, M., Huijnen, V., and Krol, M. C.: The European aerosol budget in 2006, Atmos. Chem. Phys., 11, 1117-1139, doi:10.5194/acp-11-1117-2011, 2011.

Adachi, K. and Buseck, P. R.: Internally mixed soot, sulfates, and organic matter in aerosol particles from Mexico City, Atmos. Chem. Phys., 8, 6469-6481, doi:10.5194/acp-8-6469-2008, 2008.

Adachi, K., Chung, S. H., Friedrich, H., and Buseck, P. R.: Fractal parameters of individual soot particles determined using electron tomography: Implications for optical properties, J. Geophys. Res., 112, D14202, doi:10.1029/2006JD008296, 2007.

Andersson, C., Langner, J., and Bergström, R.: Interannual variation and trends in air pollution over Europe due to climate variability during 1958-2001 simulated with a regional CTM coupled to the ERA40 reanalysis, Tellus, 59B, 77-98, 2007.

Bond, T. C.: Can warming particles enter global climate discussions?, Env. Res. Lett., 2, 045030, 2007.

Bond, T. C. and Bergstrom, R. W.: Light absorption by carbonaceous particles: An investigative review, Aerosol Sci. Technol., 40, 27-67, 2006.

Bond, T. C., Habib, G., and Bergstrom, R. W.: Limitations in the enhancement of visible light absorption due to mixing state, J. Geophys. Res., 111, D20211, doi:10.1029/2006JD007315, 2006.
Chang, H. and Charalampopoulos, T. T.: Determination of the wavelength dependence of refractive indices of flame soot, Proc. R. Soc. Lnd. A, 430, 577-591, 1990.

Draine, B. T.: The discrete dipole approximation for light scattering by irregular targets, in: Light Scattering by Nonspherical Particles, edited by Mishchenko, M. I., Hovenier, J. W., and Travis, L. D., Academic Press, San Diego, CAi USA, 131-144, 2000.

Hansen, J. and Nazarenko, L.: Soot climate forcing via snow and ice albedos, Proc. Natl. Acad. Sci., 101, 423-428, 2004.

Henyey, L. C. and Greenstein, J. L.: Diffuse radiation in the galaxy, Astrophys. J., 93, 70-83, 1941.

Jacobson, M. Z.: Strong radiative heating due to the mixing state of black carbon in atmospheric aerosols, Nature, 409, 695-697, 2001.

Kahnert, M.: On the observability of chemical and physical aerosol properties by optical observations: Inverse modelling with variational data assimilation, Tellus, 61B, 747-755, 2009.

Kahnert, M.: Modelling the optical and radiative properties of freshly emitted light absorbing carbon within an atmospheric chemical transport model, Atmos. Chem. Phys., 10, 1403-1416, doi:10.5194/acp-10-1403-2010, 2010a.

Kahnert, M.: On the discrepancy between modelled and measured mass absorption cross sections of light absorbing carbon aerosols, Aerosol Sci. Technol., 44, 453-460, 2010b.

Kahnert, M.: Numerically exact computation of the optical properties of light absorbing carbon aggregates for wavelength of $200 \mathrm{~nm}-12.2 \mu \mathrm{m}$, Atmos. Chem. Phys., 10, 8319-8329, doi:10.5194/acp-10-8319-2010, 2010c.

Koch, D. and Del Genio, A. D.: Black carbon semi-direct effects on cloud cover: review and synthesis, Atmos. Chem. Phys., 10, 7685-7696, doi:10.5194/acp-10-7685-2010, 2010.

Kondo, Y., Sahu, L., Moteki, N., Khan, F., Takegawa, N., Liu, X., Koike, M., and Miyakawa, T.: Consistency and traceability of black carbon measurements made by laser-induced incandescence, thermal-optical transmitance, and filter-based photoabsorption techniques, Aerosol Sci. Technol., 45, 295-312, 2011.

Liu, L., Mishchenko, M. I., and Arnott, W. P.: A study of radiative properties of fractal soot aggregates using the superposition $T$ matrix method, J. Quant. Spectrosc. Radiat. Transfer, 109, 26562663, 2008.

Mackowski, D. W.: Calculation of total cross sections of multiplesphere clusters, J. Opt. Soc. Am. A, 11, 2851-2861, 1994.

Mackowski, D. W. and Mishchenko, M. I.: Calculation of the T matrix and the scattering matrix for ensembles of spheres, J. Opt. Soc. Am. A, 13, 2266-2278, 1996.

Mie, G.: Beiträge zur Optik trüber Medien, speziell kolloidaler Metallösungen, Ann. Phys., 25, 377-445, 1908.

Ramanathan, V. and Carmichael, G.: Global and regional climate changes due to black carbon, Nature Geosci., 1, 221-227, 2008.

Ramanathan, V., Crutzen, P. J., Kiehl, J. T., and Rosenfeld, D.: Aerosols, Climate, and the Hydrological Cycle, Science, 294, 2119-2124, 2001.

Sorensen, C. M. and Roberts, G. M.: The prefactor of fractal aggregates, J. Colloid. Interface Sci., 186, 447-452, 1997.

Stamnes, K., Tsay, S.-C., Wiscombe, W., and Jayaweera, K.: Numerically stable algorithm for discrete-ordinate-method radiative transfer in multiple scattering and emitting layered media, Appl. Opt., 27, 2502-2509, 1988. 
Thomas, G. E. and Stamnes, K.: Radiative transfer in the atmosphere and ocean, Cambridge University Press, Cambridge, 1999.

van de Hulst, H. C.: Light Scattering by Small Particles, John Wiley \& Sons, Inc., New York, USA, p. 70, 1957.

van Poppel, L. H., Friedrich, H., Spinsby, J., Chung, S. H., Seinfeld, J. H., and Buseck, P. R.: Electron tomography of nanoparticle clusters: Implications for atmospheric lifetimes and radiative forcing of soot, Geophys. Res. Lett., 32, L24811, doi:10.1029/2005GL024461, 2005.
Wriedt, T., Wilkens, J., and Hellmers, J.: Differentiating between sintered and non-sintered aggregates, in: Electromagnetic and Light Stattering XII, edited by: Muinonen, K., Penttilä, A., Lindqvist, H., Nousiainen, T., and Videen, G., University of Helsinki, Helsinki, Finland, 322-325, 2010. 